\title{
Methanolysis of Succinic Anhydride and Some Substituted Succinic Anhydrides
}

\author{
EN DRE B ER NER and UN N K EN NERUD
}

Universitetets kjemiske institutt, Blindern-Oslo, Norway

\begin{abstract}
The velocity constant of the reaction between succinic and some substituted succinic anhydrides and methanol have been measured by means of a titration method. A definite influence of the substituents on the velocity constant was ascertained. Even in very dilute aqueous solutions of methanol a pronounced Schotten-Baumann type reaction takes place when dissolved anhydrides are titrated with alkali.
\end{abstract}

$\mathrm{T}_{\mathrm{i}, \mathrm{s}}$ he reaction between alcohols and the anhydrides of dicarboxylic acids leading to half-esters of the acids has been known for a long time. Among others Veibel and Pedersen ${ }^{1}$ studied the reaction using sodium or potassium alcoholates instead of the free alcohols, paying special interest to the proportion of isomeric esters occurring when unsymmetrically substituted anhydrides were used.

Some time ago we started an investigation in order to measure the velocity of the alcoholysis of succinic anhydride and substituted succinic anhydrides. It was first tried to measure the rate of reaction by a physical method, viz. by measuring the changes in refractive index by means of an interferometer. We did not, however, succeed in establishing satisfactory experimental conditions for such measurements and therefore turned over to use alkalimetric titrations.

Several attempts have earlier been made to determine the rate of alcoholysis of acid anhydrides by titration. Siegel and Moran ${ }^{2}$ used maleic anhydride and a number of different alcohols. The anhydride was dissolved in acetone, an alcohol added and titration carried out immediately with a solution of potassium hydroxide in ethanol. The titration showed that a complete formation of half-ester had taken place, and they drew the conclusion that maleic anhydride and an alcohol reacted instantaneously and quantitatively as in the case of ion reactions.

Their results were, however, later repudiated by Anderson and Kenyon ${ }^{3}$ and by Lavine and Herkness. ${ }^{4}$ By evaporating a solution of phthalic anhydride 
in ethanol which had been left for one hour at room temperature Anderson and Kenyon obtained only unchanged phthalic anhydride. They concluded therefore that no instantaneous reaction took place between anhydride and alcohol. Lavine and Herkness titrated solutions of anhydride in alcohol with an alcoholic as well as with an aqueous solution of potassium hydroxide. From the difference between the two titrations they meant to be able to calculate the amount of anhydride which had reacted with the alcohol. In order to avoid the influence of the alcohol during the titrations with aqueous alkali the solutions were diluted 20 times with water before titration. They drew the conclusion from their measurements that the reaction between the anhydrides and the alcohols used by them had taken place within $24 \mathrm{~h}$, but no rates of reaction were given.

As soon as we started titrations of anhydrides in alcohols it became evident that the disturbing effect of the alkali was much more prominent than supposed by Lavine and Herkness. This is in accordance with the results of Caudri ${ }^{5}$ who studied the titration of acetic anhydride in mixtures of ethyl or methyl alcohol and water. He found that the acid anhydride in the presence of alkali reacted with alcohols in aqueous solution in a similar way as do the acid chlorides in the well-known Schotten-Baumann reaction.

In order to find out to what degree this Schotten-Baumann type reaction actually would affect the titrations we made the following experiments. In a number of flasks were placed $2 \mathrm{ml} 0.1 \mathrm{~N}$ sodium hydroxide, in one of them $21 \mathrm{ml}$ water and in the other the same volume of aqueous solutions of methanol of varying concentrations. To each flask then $2 \mathrm{ml}$ of a $0.1 \mathrm{M}$ solution of succinic anhydride in anhydrous acetone were added and the solution immediately titrated with $0.1 \mathrm{~N}$ sodium hydroxide. The temperature was kept at $18^{\circ} \mathrm{C}$. The results are shown in Table 1.

As will be seen even with the very low concentration of 0.2 vol. $\%$ methanol the formation of half-ester is more than $10 \%$. This result is in itself interesting as it demonstrates the very strong nucleophilic character of the methoxyl ion. It ought to be added that the effect of the ethoxyl ion was considerably smaller. Thus by using a solution containing one vol. \% ethanol the amount of halfester formed during the titration was only $5.4 \%$ as compared with $42.5 \%$ in the case of methanol.

Table 1. Titration of aqueous solutions of succinic anhydride containing methanol.

\begin{tabular}{ccc}
$\begin{array}{c}\text { Methanol } \\
\text { in volume } \%\end{array}$ & $\begin{array}{c}\text { Consumed ml } \\
0.1 \mathrm{~N} \mathrm{NaOH}\end{array}$ & $\begin{array}{c}\text { Half-ester } \\
\text { formed in \% }\end{array}$ \\
\hline 0 & & \\
0.2 & 4.000 & 0 \\
0.4 & 3.750 & 12.5 \\
1 & 3.431 & 28.5 \\
2 & 3.151 & 42.5 \\
4 & 2.831 & 58.5 \\
8 & 2.605 & 69.8 \\
10 & 2.394 & 80.3 \\
20 & 2.349 & 82.6 \\
\end{tabular}


Being now acquainted with the Schotten-Baumann type effect mentioned above it was possible to work out a method for alkalimetric determination of the rate of alcoholysis of the acid anhydrides. A freshly prepared solution of the anhydride in anhydrous alcohol was kept at constant temperature. At certain times samples were withdrawn, diluted with water and left standing for one hour, in which time the unreacted anhydride was found to hydrolyse completely. The solutions, now containing only free acid and half-ester, could easily be titrated. Due to the fact that the acid anhydride reacts somewhat with the diluted alcohol even without the presence of alkali, viz. during the hour in which the solution was left standing after dilution, a correction had to be applied to the observed values. These corrections were determined as described in the experimental part. The rate of the reaction was calculated from the equation

$$
k_{t}=\frac{2.303}{t} \log \frac{100}{100-x_{t}}
$$

in which $x_{t}$ is the corrected concentration of half-ester in per cent at the time $t$. The results are collected in Table 2 .

As will be seen the effect of introducing an alkyl group is to increase the rate of alcoholysis: in the case of one methyl group to double the rate. This is the opposite of what should be expected from the fact that a methyl group has a positive inductive effect. The explanation of the increase in rate must obviously be that the introduction of a methyl group in succinic anhydride makes the molecule unsymmetric, and that such an unsymmetric molecule reacts more readily than a symmetric one. When another methyl group is introduced such as in mesodimethylsuccinic anhydride, the molecule again becomes symmetric and the influence of the substituents on the rate is then insignificant. On the other hand, when an electron accepting substituent such as halogen is introduced, the rate of alcoholysis is as expected increased considerably; by chlorine nearly 200 times.

\section{EXPERIMENTAL}

Preparation of the anhydrides. Succinic anhydride was obtained from the acid in the usual way by treatment with phosphorus oxychloride. It was purified by distillation and subsequent crystallizations from chloroform. In some cases it was finally sublimated; m.p. $117-118^{\circ}$.

Table 2. Rate of methanolysis of some anhydrides of dicarboxylic acids at $23^{\circ} \mathrm{C}$.

\begin{tabular}{|c|c|c|c|}
\hline Anhydride & $k^{23 \circ}$ & \multicolumn{2}{|c|}{ Half life } \\
\hline Succinic anhydride & 0.00180 & $6 \mathrm{~h} 25$ & $\min$ \\
\hline Methylsuccinic anhydride & 0.00369 & $3 \gg 8$ & $"$ \\
\hline Ethylsuccinic anhydride & 0.00268 & $4 \geqslant 16$ & " \\
\hline Mesodimethylsuccinic anh. & 0.00208 & $6 \gg 32$ & $"$ \\
\hline Monochlorosuccinic anh. & 0.33 & 2.1 & " \\
\hline Monobromosuccinic anh. & 0.118 & 5.9 & " \\
\hline
\end{tabular}

Acta Chem. Scand. 17 (1963) No. 10 
Methylsuccinic anhydride was prepared from the acid and purified as described by Berner and Leonardsen; ${ }^{6}$ m.p. $69.5^{\circ}$.

The racemic ethylsuccinic acid was prepared according to the method of Polko; m.p. 98-99 ${ }^{\circ}$. The corresponding anhydride, prepared as described by Berner and Leonardsen $^{6}$ for the optically active anhydride, was a liquid and could therefore only be purified by repeated distillations in a vacuum. (Equiv. wt: Found 64.6. Calc. 64.1).

The mesodimethylsuccinic acid was a by-product left over from Berner and Leonardsens preparation of the corresponding racemic acid. ${ }^{6}$ The meso-anhydride was obtained from the acid by treatment with acetyl chloride. Recrystallized from benzene; m.p. $43^{\circ}$.

Monochlorosuccinic acid was prepared from malic acid according to Walden. ${ }^{8}$ It was recrystallized by dissolving in a small quantity of ether and adding benzene; m.p. 150$152^{\circ}$. The conversion of the acid into the anhydride was carried out as described by Anschütz and Bennert. ${ }^{\circ}$ Purified by distillation in a vacuum; m.p. $40-41^{\circ}$.

A commercial product of monobromosuccinic acid was converted into the anhydride as described for the chloro-acid. Some difficulties were encountered in obtaining a crystalline product, but after keeping the anhydride for a few days at $-18^{\circ} \mathrm{C}$ it crystallized. Dried in a vacuum above phosphorus pentoxide it had m.p. $30^{\circ}$.

It was found essential that the methanol should be as free from water as possible. The methanol was therefore treated two, in some cases even three times with metallic magnesium as described by Bjerrum and Zechmeister. ${ }^{10}$ After such a treatment the methanol gave no precipitate with lead tetracetate, but only a light yellow colour, indicating the content of water to be less than $10 \mathrm{mg}$ per $100 \mathrm{ml}$.

The titrations. In our earliest experiments we followed Lavine and Herkness ${ }^{4}$ in that we first dissolved the anhydride in a small amount of anhydrous acetone and then added methanol to the desired volume. Although this procedure allowed the exact definition of zero time in an experiment, it was found to have draw-backs so that we later on dis. solved the anhydride directly in the methanol. The greatest advantage of this procedure was that, at the end of the experiments, we found values which showed $100 \%$ formation of the half-ester.

As an example some details from an experiment with succinic anhydride are given. For the other anhydrides the procedure was quite similar. Pulverized and well dried succinic anhydride $(0.505 \mathrm{~g})$ was dissolved in methanol to a volume of $50 \mathrm{ml}$ and the solution then kept in a thermostat at $23^{\circ} \mathrm{C}$. At certain times samples of $2 \mathrm{ml}$ were withdrawn, diluted with $50 \mathrm{ml}$ distilled water, left standing for one hour and then titrated with $0.1 \mathrm{~N}$ sodium hydroxide. As the original concentration of succinic anhydride was $0.101 \mathrm{M}$, the anhydride in $2 \mathrm{ml}$ solution would require $4.04 \mathrm{ml}$ of the alkali if no reaction with methanol had taken place. With a $100 \%$ formation of half-ester $2.02 \mathrm{ml} 0.1 \mathrm{~N} \mathrm{NaOH}$ would be required. If the requirement of alkali was found to be $V_{t}$ at the time $t$, the percentage of halfester at that time would be $100\left(4.04-V_{t}\right) / 2.02$.

As already mentioned it was necessary to apply a correction to the results of the titrations because a certain reaction between anhydride and methanol took place even

Table 3. Methanolysis of succinic anhydride at $23^{\circ} \mathrm{C}$.

\begin{tabular}{|c|c|c|c|c|}
\hline Time & $\begin{array}{l}0.1 \mathrm{~N} \mathrm{NaOH} \\
\text { in } \mathrm{ml}\end{array}$ & \multicolumn{2}{|c|}{ Half-ester in percent } & $\begin{array}{c}\text { Velocity } \\
\text { constant } k \times 10^{3}\end{array}$ \\
\hline $23 \mathrm{~min}$ & 3.74 & 14.9 & 4.6 & \\
\hline $32 》$ & 3.69 & 17.3 & 7.3 & \\
\hline $95 \gg$ & 3.53 & 25.2 & 16.2 & 1.86 \\
\hline $120 "$ & 3.46 & 28.7 & 20.1 & 1.87 \\
\hline $155 \gg$ & 3.35 & 34.2 & 26.2 & 1.96 \\
\hline $195 »$ & 3.32 & 35.6 & 27.8 & 1.67 \\
\hline $225 \gg$ & 3.26 & 38.6 & 31.1 & 1.55 \\
\hline 250 & 3.18 & 42.6 & 35.5 & 1.66 \\
\hline $24 \mathrm{~h}$ & 2.18 & 92.1 & 91.1 & \\
\hline $72 》$ & 2.02 & 100 & 100 & \\
\hline
\end{tabular}


Table 4. Velocity constants at $23^{\circ} \mathrm{C}$ for various anhydrides.

\begin{tabular}{llll}
\hline \multicolumn{1}{c}{ Anhydride } & $\begin{array}{c}\text { Velocity constants } \\
\text { means from each experiment }\end{array}$ & Overall mean \\
\hline Succinic anhydride & $1.74, \quad 1.94, \quad 1.96, \quad 1.76$ & $1.80 \times 10^{-3}$ \\
Methylsuccinic anhydride & $3.73, \quad 3.69, \quad 3.65$ & 3.69 " \\
Ethylsuccinic anhydride & $2.63, \quad 2.52, \quad 2.91$ & 2.68 & " \\
Mesodimethylsuccinic anh. & $1.96, \quad 2.26, \quad 2.03$ & 2.08 \\
Monochlorosuccinic anh. & $0.307,0.331,0.306$ & 0.33 \\
& $0.359,0.353$ & 0.118
\end{tabular}

after the dilution with water. The concentration of methanol after dilution was about 4 vol. $\%$ and there was a great surplus of methanol, the molecular fraction being about 200. A series of experiments in which solutions of succinic anhydride in $4 \%$ methanol were titrated showed $(i)$ that the anhydride had reacted completely within one hour and $(i i)$ that the fraction of half-ester formed was independent of the concentration of the anhydride when this was varied between 0.002 and $0.008 \mathrm{M}$. The amount of halfester formed when pure succinic anhydride was dissolved in $4 \%$ methanol was found to be $10.8 \%$. Calling this correction factor $a$, the corrected value $x_{t}$ corresponding to the value $V_{t}$ calculated from the titration at the time $t$ can be calculated from the formula

$$
x_{t}=V_{t}-\left(100-x_{t}\right) \frac{a}{100}
$$

In Table 3 are given the results from one of the experiments with succinic anhydride. As can be expected the accuracy is not high and for that reason the first and the last observations have not been used in calculating the velocity constant $k$ from the following equation:

$$
k_{t}=\frac{2.303}{t} \cdot \log \frac{100}{100-x_{t}}
$$

The mean value for $k$ from the above experiment is $1.74 \times 10^{-3}$. In three other experiments with succinic anhydride the mean values given in Table 4 were found, thus giving an overall mean of $1.80 \times 10^{-3}$.

Also for the other anhydrides two or more experiments were carried out, but the details are not included here. Instead the mean velocity constant for each experiment and the overall mean for each anhydride are given in Table 4.

The earliest part of this investigation was carried out by cand. real. Kari Koller. One of us (U.K.) is indebted to Alf Bjerkes legat for a grant.

\section{REFERENCES}

1. Veibel, S. and Pedersen, Chr. Acta Chem. Scand. 9 (1955) 1674.

2. Siegel, E. F. and Moran, M. K. J. Am. Chem. Soc. 69 (1947) 1457.

3. Anderson, I. G. and Kenyon, J. Ibid. 70 (1948) 3952.

4. Lavine, T. F. and Herkness, C. Ibid. 70 (1948) 3951.

5. Caudri, J. F. M. Rec. Trav. Chim. 48 (1929) 778; 49 (1930) 1.

6. Berner, E. and Leonardsen, R. Ann. 538 (1939) 1.

7. Polko, G. Ann. 242 (1887) 114.

8. Walden, P. Ber. 26 (1893) 214.

9. Anschütz, R. and Bennert, C. Ber. 15 (1882) 643.

10. Bjerrum, N. and Zechmeister, L. Ber. 56 (1923) 894.

Received September 4, 1963. 